\title{
Evolution of Instruments in Endodontics-Literature Review
}

\author{
Andrea Gisella Flores ${ }^{1,{ }^{*}}$, and Aissa Pastenes Orellana ${ }^{2}$ \\ ${ }^{1}$ University of San Martin de Porres, Endodontics Specialist, Inca Garcilaso de la Vega University, Peru \\ ${ }^{2}$ University of Chile, Endodontics Specialist, Peruvian University Cayetano Heredia, Peru
}

*Corresponding author: Andrea Gisella Flores, University of San Martin de Porres, Endodontics Specialist, Inca Garcilaso de la Vega University, Peru, E-mail: andreaflores30@hotmail.com

Received: 03 Sep, 2019 | Accepted: 14 Oct, 2019 | Published: 21 Oct, 2019

Citation: Flores AG, Pastenes A (2019) Evolution of Instruments in Endodontics-Literature Review. Int J Dent Oral Health 5(6): dx.doi. org/10.16966/2378-7090.306

Copyright: (C) 2019 Flores AG, et al. This is an open-access article distributed under the terms of the Creative Commons Attribution License, which permits unrestricted use, distribution, and reproduction in any medium, provided the original author and source are credited.

\section{Abstract}

The chemomechanical preparation is an essential part of the root canal therapy; it is the sum of auxiliary chemical substances and endodontic instruments suitable to achieve adequate cleaning, modeling and disinfection of the root canals. There is a huge evolution of manual and mechanized instruments in order to achieve the biological and technical objectives of the endodontic therapy.

The intricate anatomy of the root canals makes cleaning and shaping difficult, which is why the introduction of nickel titanium alloy in the fabrication of endodontic files with its super elastic properties and memory effect has been transcendental to better address narrow, curved and difficult to shape canals.

The purpose of this literature review article is to emphasize concepts and highlight positive and vulnerable aspects in the evolution of endodontic instruments.

Keywords: Endodontic; Root canal preparation; Evolution of endodontic instruments; Nickel titanium alloys

\section{Introduction}

The importance of chemomechanical preparation is extreme, that is why researchers have focused on the fulfillment of technical and biological objectives of pulp therapy. In the last two decades, the evolution of manual and mechanized instruments has contributed to improve the success and predictability of the endodontic treatment [1-4].

The purpose of this literature review article is to emphasize concepts and highlight positive and vulnerable aspects in the evolution of endodontic instruments. The qualitative method of direct type content analysis was used; the information review was based on the search information in journals indexed to Medline, Scielo, PubMed, Embase, Lilacs; with the use of descriptors such as: "endodontics", "root canal preparation", "evolution of instruments in endodontics", "NiTi alloys". The inclusion criteria were: languages: Spanish, English and Portuguese; any geographic area or country preferably published in the last five years and the manual search of books and magazines was also considered. The Exclusion Criteria were: clinical cases and reports. This entire search in order to allow the clinician the best choice in terms of evolution of instruments in endodontic to achieve a better preparation based on scientific evidence.

In 1838 Edward Maynard created the first endodontic instrument starting with the spring of a clock with the objective of cleaning and widening the root canal [1-5].
The first endodontic files were made of carbon steel, whose hardness greater than dentin, prevent its use in curved canals and could cause iatrogenies such as: deformation of the apical foramen, anatomical deviation of the canal and perforations, that is due to the greater filing on the curved wall. They were manufactured without scientific criteria. Among its advantages, resistance to fracture and high cutting capacity could be highlighted, but diminished by being highly corrosive due to the joint action of chemical substances such as sodium hypochlorite, negatively affecting the resistance of the file $[6,7]$.

The replacement of carbon steel for stainless steel instruments used today solved the corrosion problem. In 1955 John Ingle, a professor at the University of Washington USA, introduced the standardization of instruments; together with Levine in 1961. They published the first work on standardized instruments, as well as the gutta-percha and silver cones accordingly $[4,6,7]$.

The A.A.E. accepted the proposal of Ingle and Levine by altering its lightly and resulting to what is now known as International Standard Organization (ISO). In 1976 it was the American Standardization Association who approved Specification No. 28 which presents the standards for the manufacture of files and reamers $[4,8]$.

In 1981, after 26 years of studies the final norms of Specification No. 28 were disclosed, and the current international standardization 
of the instruments was then defined. The Kerr Manufacturing Co. Industry was the first to build these instruments that were known as K-type instruments, also being the most copied in the world $[4,8]$.

The active part of an instrument starts at the tip and is called D1, and ends at part D2 $[4,8]$.

The diameter D2 in the active part must measure 0.32 more than the diameter D1, since the taper increase from D1 to D2 is 0.02 $\mathrm{mm}$ per $\mathrm{mm}$ of the active part and its extension must be at least 16 $\mathrm{mm}[4,8]$.

The total lengths of the instruments are $21,25,28$ and $31 \mathrm{~mm}$, the color coding on the handles facilitates their identification $[4,8]$.

The characteristics of manual instruments can be summarized as follows: Made up of stainless steel, colored plastic handle and the increase of the standard taper of $0.02 \mathrm{~mm}$ per $\mathrm{mm}$ in the active part $[4,8]$.

The stainless steel, which is still used today, presents little flexibility which affects the instrumentation in curved canals, producing iatrogenic errors such as root canal deviation, foramen dislocation, and root perforation, among others. This occurs because the instrument, being rigid causes great wear on the curved wall (considered danger zones). That is why, after the discovery of the NiTi alloy, it was introduced in dentistry $[4,9]$.

It was discovered by the metallurgical engineer William Buehlerin 1963, he named it NiTiNOL because of the elements of the alloy: Nickel (Ni), Titanium (Ti) and the name of the laboratory where it was produced (NOL) Navy Ordnance Laboratory in Maryland, United States $[7,10]$.

It belongs to the group of alloys with special properties characterized by the effect of Shape memory and Super elasticity.

This very unique and useful alloy has shape memory, that is, it can return to its previous shape, it also has corrosion resistance, great biocompatibility and a reasonable cost $[4,7,10]$.

Due to the great clinical importance and the impact of its discovery, we will give a special description and written coverage on this alloy. Leonardo and Leal in 1998 considered the creation of this as the greatest technological evolution for the preparation of root canals, especially in molars $[10,11]$.

The equiatomic NiTiNOL alloy, containing 56\% nickel and 44\% titanium, may currently vary by manufacturer $[4,10,12]$.

Mc Spadden in 1993 took advantage of the properties of super elasticity and shape memory of this alloy, creating instruments that can rotate inside of the root canal at 360 degrees, originating the first mechanical system. Although these instruments changed endodontic instrumentation, another problems associated with files fracture surfaced $[4,10-14]$

\section{Manufacturing of Endodontic Instruments}

They are manufactured in two ways:

\section{Torsion}

It occurs when the circular section wire, usually stainless steel, undergoes wear on its sides resulting in flat surfaces that will produce the future section of the file. Usually these sections can be quadrangular, triangular or rhomboid. After having achieved the section, the wire is held at its ends and twisted to obtain the turns of the active part [8].

\section{Wear}

The wear happens as follows: the circular section wire is introduced in two lathes that generate wear of the wire, producing the desired sectional configuration. It is important to note that files manufactured by wear tend to be less resistant than files manufactured by torsion, as wear causes mass loss, which consequently reduces resistance.

In its manufacturing process, stainless steel files accept both manufacturing processes well, unlike NiTi files which must be manufactured with the wear process due to their special shape memory effect and super elasticity. Allowing permanent deformation of the metal $[8,10]$.

\section{What are the causes and prevention of fractures of NiTi} instruments?

The most common accident in the use of nickel titanium instruments is fracture, which determines a great difficulty in removing a separate instrument from inside the root canal. These causes of separation or fracture of the instruments can be grouped in clinical and metallographic form [10].

Clinical causes: Lack of previous elimination of cervical and coronal interferences: The elimination of interferences constitutes an indispensable aspect in the preparation of the root canal either using stainless steel or nickel titanium instruments to access in a direct way to the canal [13].

Prior patent absent: It is a requirement to carry out the previous probing or catheterization with stainless steel manual $\mathrm{K}$ files in the segment of the root canal that will be subjected to the action of the rotary instruments, in this way the difference between the diameter of the tip is reduced or equalized of the instrument and that of the duct. That is, mechanized instruments are preceded by manual instruments $[4,9,12]$.

Metallographic causes: Inadequate Kinematics in NiTi instruments. They occur when the instrument rotates inside of the root canal and suffers two types of stress or fatigue, by torsion and flexion $[4,9,12]$.

Torsional fatigue: It occurs when a segment of the instrument, usually the tip locks in the walls of the root canal and is immobilized without being able to overcome the resistance of the dentin to produce the cut; The other end of the instrument is subjected to the axial torque generated by the motor when turning. A stress is then created that exceeds the limit of elasticity of the metal, producing a plastic deformation followed by the unwanted fracture of the instrument $[8,15,16]$.

In summary, the fracture occurs if the section of the canal is smaller than the tip of the instrument impeding to cut the dentin $[4,10,17]$.

Flexural fatigue: Flexural stress is directly related to the curvature of the root canals, the diameter of the instrument and the speed of rotation, which are variables that determine the lifespan of the instrument $[17,18]$.

Cyclic fatigue is the altered repetition of tension-compression cycles on a point of the instrument when it rotates in a curved root canal. An instrument with less conicity will with stand flexural stress better than an instrument with greater conicity $[17,19]$.

\section{Generations of the mechanized systems according to their alloy}

Classification according to Leonardo MR and de Toledo Leonardo $\mathrm{R}[12]$. 
First generation rotating systems $(\mathrm{NiTi})$ : They are mechanized instruments manufactured with NiTi Alloy, that is, they have the special properties of super elasticity and shape memory. This generation of files offers superior quality in terms of flexibility and strength. The most used are: Quantec System (Tycon/Analytic Endod/Kerr-United States), ProFile System 04/.06 (Dentsply/Maillefer-Switzerland), K3 Endo System (SDS Kerr-Sybron Endo-United States), Mtwo ${ }^{\oplus}$ System (VDW Dental-Industry), ProTaper ${ }^{\circledR}$ Original (Dentsply/MailleferSwitzerland), ProTaper Universal (Dentsply/Maillefer-Switzerland) $[4,11,17,20]$.

- Advantages: Optimum flexibility is the ability of the instrument to accompany the curvature of a root canal avoiding the formation of steps, perforations along the root canal or at the apical level; cutting efficiency; reduced working time for the operator, greater comfort for the patient and a lower incidence of post operative pain $[4,11,17,20]$.

- Disadvantages: They generate costs for the operator since they must be used only once; possibility of instrument fracture; poor cleaning in flattened root canals $[4,11,17,20]$.

Second generation rotating systems (NiTi Phase R): Instruments manufactured with NiTi Phase R Alloy (Rombiodal), appear with the objective of improving the properties of the first generation alloys files, increasing their flexibility and reducing the risk of fracture, being manufactured by different thermal processes and also by torsion $[4,8,12,13]$.

The intermediate phase (R) enable the instrument to be twisted by creating stretch marks and cutting teeth without causing microfractures, giving the instrument greater flexibility and resistance to torsional fracture $[4,8,12]$.

Within the most used systems we have: K3 XF (Sybron EndoKavo/Kerr) Phase R/Wear, Twisted File (League R-Twisted), Easy Shaper (Phase R-Easy Dental Equipment-Belo Horizonte, MG, Brazil) $[4,12,13]$.

- Advantages: High resistance to cyclic and torsional fatigue, lower fracture index, greater cutting efficiency, offers a shorter working time providing a reduction of stress for the professional, lower incidence of post-operative pain, therefore in greater comfort for the patient $[4,10-13]$.

- Disadvantages: They generate costs to the operator since they must only be used once, possibility of fracture but with a lower incidence than with the first generation instruments [4,10-13].

The NiTi Alloy Phase R was also used in the manufacture of the Oscillating (Asymmetric) Systems, usually called Reciprocants [4,10-13].

Oscillating Systems (Reciprocants)

TF Adaptive (2013) (Sybron Endo Kerr Group) [4,10,12,13].

Third generation rotating systems: ( $\mathrm{NiTi}, \mathrm{M}-\mathrm{Wire})$ :

Instruments manufactured with this alloy: ProFile ${ }^{\star} \mathrm{GT}$ (Great Tapers), Series X (NiTi M-Wire) (Dentsply/Maillefer-Switzerland), ProFile $^{\infty}$ Vortex $^{\text {tu }}$ (NiTi M-Wire) (Dentsply Tulsa/Dental-United States), Protaper Next (NiTi M-Wire) (Dentsply Tulsa/Dental-United States).

They have a lower modulus of elasticity than instruments made of NiTi with conventional alloys, which is why they are highly flexible.

This new thermal processing technology optimizes the microstructure of nickel titanium alloys $[8,9,12]$.
The NiTi alloy with M-Wire Treatment is also used in the manufacture of a (single) ProGlider ${ }^{\mathrm{rax}}$ (Dentsply/Sirona-United States) rotary instrument, intended to perform the Glide Path (prepreparation) of straight, curved or atresic ducts for then continue with instrumentation using rotary or oscillatory systems.

Other instruments made with NiTi (M-Wire) are the Oscillatory or Reciprocant Instruments $[8,9,12]$.

Reciprocant systems: Wave One (Dentsply/Maillefer-Switzerland), Reciproc (Dentsply/VDW-Germany) [3,5,21].

Shen Y, et al. [5], conducted a study on the defects of 458 Wave one instruments (Small, Primary and large) after a single use in different endodontic clinics. Not only did they verify the effectiveness of the M-wire alloy in terms of the flexibility of the instruments, but it was also demonstrated that the reciprocating movement extends the lifespan of NiTi instruments and their resistance to fatigue compared to continuous rotation.

Wave One uses a single instrument per conduct, being able to perform a good conformation of the root canals. They concluded that the risk of fracture occurs mostly with Wave One Small $[3,5,21]$.

- Advantages: High resistance to cyclic and torsional fatigue, lower fracture index when used more than once, greater cutting efficiency, greater flexibility compared to first generation (15\% more flexible), less working time for the professional, greater comfort for the patient, lower incidence of post operative pain $[3,5,21]$.

- Disadvantages: They generate higher costs, because according to the manufacturer they must be used only once; possibility of fracture, but with a lower incidence compared to the instruments of the previous generation $[3,5,21]$.

Fourth generation rotating systems (NiTi CM): The fourth generation systems have nickel MC titanium alloys (memory control). Coltene (United States), in 2012 used this alloy to manufacture the HyFlex System.

Easy Dental Equipment (Bello Horizonte, MG, and Brazil) in 2014 also used the Nickel Titanium MC alloy, using the Easy Pro Design Logic System. They have an extreme flexibility, so they are indicated in root canals with severe curvatures, performing the action of "meandering" through the light of the root canal $[1,4,8,12]$.

Recently Coltene (United States) launched the HyFlex ${ }^{\mathrm{TM}} \mathrm{EDM}$ NiTi Files System (Electrical Discharge Machining) to the endodontic market. They are the first endodontic files manufactured by a process known as electro-erosion or Electrical Discharge Machining (EDM), consisting of the formation of a material by removing particles from its surface, using pulsating electric current; The electrical sparks cause a local melt on the surface and due to a partial evaporation of the material, the typical finish is a rough surface with regularly distributed craters. This process is used in engineering to manufacture difficult-toconform parts with conventional techniques $[1,4,12,18,21]$.

Pereira ÉS, et al. Demostrated that the new EDM process had a significant impact on the lifespan of Hyflex ${ }^{\mathrm{TM}}$ EDM files, since they showed no surface alterations or separations after multiple in vitro uses, which confirms a high wear resistance, also concluded that the Hyflex ${ }^{\mathrm{TM}} \mathrm{EDM}$ files have a surprising resistance to cyclic fatigue, up to $700 \%$ higher than $\mathrm{HyFlex}^{\circ} \mathrm{CM}^{\mathrm{rx}}$ files, so it would be suitable instruments for shaping root canals with severe curvatures $[1,4,9,12,18,21]$. 
Instruments manufactured with NiTi CM-495 Alloy: Hyflex Coltene System (United States), Easy Pro Design System (Brazil).

Instruments manufactured with NiTi CM-495 EDM Alloy: HyFlex ${ }^{\oplus}$ EDM System.

Instruments manufactured with NiTi CM-500 Alloy: XP-Endo (FKG Dentaire-Switzerland).

- Advantages: Is indicated for root canals with severe anatomical complexity. High resistance to cyclic fatigue, lower fracture index. (300\% more resistant), greater cutting efficiency, greater flexibility, $80 \%$ more flexible than conventional instruments, offers less working time, lower incidence of post-operative pain, greater patient comfort $[1,4,9,12,18,21]$.

- Disadvantages: Contraindicated for wide or relatively wide and straight ducts $[1,4,9,12,18,21]$.

Fifth generation rotating systems (NiTi, Gold): Manufactured with nickel titanium alloy with GOLD Treatment. ProTaper GOLD (Dentsply/Sirona-United States) $[4,15,18,21]$.

- Advantages: They are $80 \%$ more flexible than the files of conventional rotary systems, more resistant to cyclic fatigue by $150 \%$ compared to the Universal Protaper System, shorter working time $[4,16,18,21]$.

This Alloy with Gold Treatment is also used in the manufacture of Reciprocant Sytems [4,15,18,21].

\section{Wave One ${ }^{\oplus}$ Gold (Dentsply/Sirona-United States) $[5,4,22]$.}

Keeping the same advantages, being even 50\% more resistant to cyclic fatigue than Wave One instruments, in terms of flexibility it is $80 \%$ higher than it predecessor Wave One $[1,4,16,18,21]$.

The Wave One ${ }^{\bullet}$ Gold system combines two important aspects in instrumentation innovation: the heat treatment of the NiTi alloy and the reciprocating movement.

This heat treatment corresponds to predetermined cycles of cooling and heating which have been patented by the manufacturer, this gives the color and name to the Wave One ${ }^{\oplus}$ Gold files, since it produces a superficial layer of titanium oxide which gives it a golden appearance $[5,4,22]$.

This process seeks to modify the crystallographic matrix of $\mathrm{NiTi}$, altering the transformation temperatures between the austenitic and martensitic phase, creating more flexible instruments, resistant to cyclic fatigue and with greater deformability $[4,16,18,21]$.

This treatment is carried out after the instrument is manufactured, unlike other heat treatments that are carried out before carving it, such as the one used to make M-Wire from Protaper Next ${ }^{\circ}$ and Reciproc ${ }^{\circ}$ (VDW) or the R-Phase of Twisted Files ${ }^{\bullet}$ (SybronEndo) $[1,4,16,18,21]$.

Wave One ${ }^{\otimes}$ Gold files are not presented as controlled memory files, such as Hyflex $\mathrm{EDM}^{\bowtie}$ (Coltene-Whaledent), but it is normal to see them with a slight curvature at their tip, thanks to the properties of the Gold alloy. This gives the clinical advantage, in relation to conventional NiTi files, of being easier to introduce to hard-to-reach canals, such as mesiovestibular $[1,4,8,21,23]$.

New generation system (NiTi, Blue): [16-18,20] They are manufactured with NiTi Alloy with Blue Treatment.

NiTi Vortex Blue ${ }^{\mathrm{mm}}$ instruments (Dentsply/Sirona-United States) manufactured with Nitinol 508 and consisting of a blue titanium oxide layer, not seen in traditional super-elastic NiTi instruments [22-24].
This relatively hard surface layer of Vortex Blue instruments can compensate the loss of hardness compared to Profile Vortex M-Wire $[11,19,22,23]$.

Vortex Blue and shape memory technologies use special thermal processes to control the transition of extreme temperatures, making the instruments highly flexible [11,16-20].

- Advantages: Indicated for root canals that present a severe anatomical complexity. They follow the natural curvature of the root canal due to its great flexibility, greater resistance to cyclic fatigue (65\% higher in relation to instruments manufactured with M-Wire ${ }^{\oplus} \mathrm{NiTi}$ and $99 \%$ in relation to the first generation NiTi instruments), greater resistance to torsion, offers a lower working time, a reduced memory effect of the instrument (that is, it can be pre-curved slightly and not return to its original position), generating a less resistance of the instrument when introduced into the root canal $[16-18,20]$.

Vortex Blue instruments have improved material properties within creases fatigue resistance and flexibility compared to Profile Vortex (M-Wire) instruments. However, both NiTi Blue and M-wire alloy show better fatigue resistance compared to conventional nickel titanium alloy. Torsion resistance is also affected by the cross-sectional design, the metal mass and the properties of the alloy $[11,19,23]$.

Shen Y, et al. [22], conducted a study where they compared the Profile Vortex (M-Wire) and Vortex Blue systems after their clinical use. Each system was used up to three times, except in very curved or calcified ducts, they were discarded at their first use. They concluded that the fracture rate in that study was too low for a difference between the two systems [24].

\section{Reciprocant Systems:}

Reciproc $^{\circledast}$ Blue (VDW-ZIPPERER): The difference between Reciproc $^{\star}$ files and Reciproc ${ }^{\bullet}$ Blue files is the improved NiTi alloy with which the latter are manufactured, since they are subjected to an innovative thermo-mechanical treatment, which modifies its molecular structure giving the instrument increased resistance to cyclic fatigue, greater flexibility, better memory control and its characteristic blue color [24-27].

Advantages: They are very flexible to penetrate even more smoothly and safer into the root canal allowing a better centering of the instrument following the natural path, more resistant to cyclic fatigue [24-27].

De-Deus G, et.al. [27], they evaluated the fatigue resistance and flexibility of Reciproc instruments with blue thermo mechanical treatment versus conventional Reciproc ${ }^{\oplus} \mathrm{M}$-Wire instruments. They concluded that Reciproc ${ }^{\circledR}$ Blue in nickel titanium showed improved performance compared to the conventional Reciproc ${ }^{\circ}$ system in terms of flexibility and fatigue resistance; also presented a reduced micro hardness maintaining similar characteristics on the surface [24-27].

\section{Conclusions}

- In the evolution of instruments in endodontics, NiTi alloy stands out; its importance is the unique ability to work curvatures in mechanized movement without undergoing permanent plastic deformation or failures.

- The memory effect and super elasticity make the NiTi alloy an ideal material for the manufacture of endodontic instruments.

- NiTi instruments reduce the operator's working time and provide greater comfort for the patient. 
- New generations of files are offered in the market to improve and enhance the properties of the conventional NiTi alloy.

- The current trend of instruments is to manufacture them with new alloys and heat treatments, reducing the ability of the alloy to have memory. The alloy in this state has been shown to better follow the trajectory of curved root canals.

So far there is no file or mechanized system considered the best, that is, none can achieve all expectations and needs as they have different characteristics. Clinical protocols and appropriate working parameters continue to be refined as new instruments continue to be introduced in the market. Currently, the clinician has a wide variety of systems to decide which one use to achieve a much more predictable outcome in every specific case.

\section{References}

1. Topçuoğlu HS, Topçuoğlu G, Akti A, Düzgün S (2016) In Vitro Comparison of Cyclic Fatigue Resistance of ProTaper Next, HyFlex $\mathrm{CM}$, OneShape, and ProTaper Universal Instruments in a Canal with a Double Curvature. J Endod 42: 969-971.

2. Ninan E, Berzins DW (2013) Torsion and Bending Properties of Shape Memory and Superelastic Nickel-Titanium Rotary Instruments. J Endod 39: 101-104.

3. Julian Webber (2015) Shaping canals with confidence: WaveOne GOLD single-file reciprocating system. Roots International Magazine of Endodontology 11: 34-40.

4. Lopes H, Siqueira J (2015) Endodontics-Biology and Technology. $4^{\text {th }}$ Edition, Elsevier Brazil 547-550.

5. Shen Y, Coil JM, Mo AJ, Wang Z, Hieawy A, et al. (2016) Wave One Rotary Instruments after Clinical Use. J Endod 42: 186-189.

6. Murray P (2015) A Concise Guide to Endodontic Procedures. Springer 137.

7. Gutmann JL, Lovdahl PE (2012) Soluções Em Endodontia-Prevenção, Identificação e Procedimentos. (5a Edition) Elsevier, Rio de Janeiro, Brazil 195-206.

8. Haapasalo M, Shen Y (2013) Evolution of nickel-titanium instruments: from past to future. Endodontic Topics 29: 3-17.

9. Lima M (2016) Endodontics-Science and Technology. Amolca editorial, Sao Paulo 195: 215-217.

10. Torabinejad M, Walton RE, Fouad A (2015) Endodontics Principles and Practice. (5a Edition) Elsevier, Saunders 220-221.

11. de Almeida BC, Ormiga F, de Araújo MC, Lopes RT, Lima IC, et al. (2015) Influence of heat Treatment of Nickel-Titanium Rotary Endodontic Instruments on Apical Preparation: A Micro-Computed Tomographic Study. J Endod 41: 2031-2035.
12. Leonardo MR, de Toledo Leonardo R (2017) Tratamento de Canais Radiculares. 2ae edicao, Artes Médicas 220-233.

13. Perdigao J (2016) Restoration of Root Canal-Treated Teeth an Adhesive Dentistry Perspective. Springer 31-32.

14. Gutiérrez JF, Cajamarca DC, Monroy JC (2015) Alteraciones de la estructura de la lima primaria posterior a su uso. Dialnet 34: 29-36.

15. Peters OA, de Azevedo Bahia MG, Pereira ES (2017) Contemporary Root Canal Preparation: Innovation in Biomechanics. Dent Clin North Am 61: 37-58.

16. Ha JH, Kim SR, Versluis A, Cheung GS, Kim JW, et al. (2015) Elastic Limits in Torsion of Reciprocating Nickel-Titanium Instruments. J Endod 41: 715-719.

17. Lopes HP, Elias CN, Vieira MV, Vieira VT, de Souza LC, et al. (2016) Influence of Surface Roughness on the Fatigue Life of NickelTitanium Rotary Endodontic Instruments. J Endod 42: 965-968.

18. Pereira ÉS, Viana AC, Buono VT, Peters OA, Bahia MG (2015) Behavior of Nickel-Titanium Instruments Manufactured with Different Thermal Treatments. J Endod 41: 67-71.

19. Plotino G, Grande NM, Cotti E, Testarelli L, Gambarini G (2014) Blue Treatment Enhances Cyclic Fatigue Resistance of Vortex NickelTitanium Rotary Files. J Endod 40: 1451-1453.

20. Higuera O, Plotino G, Tocci L, Carrillo G, Gambarini G (2015) Cyclic Fatigue Resistance of 3 Different Nickel-Titanium Reciprocating Instrumens in Artificial Canals. J Endod 41: 913-915.

21. Steinfort K (2017) WaveOne ${ }^{\circledR}$ Gold: La nueva apuesta de Dentsply Sirona. Revista de la Sociedad de Endodoncia de Chile N 35.

22. Shen Y, Zhou H, Coil JM, Aljazaeri B, Buttar R, et al. (2015) ProFile Vortex and Vortex Blue Nickel-Titanium Rotary Instruments after Clinical Use. J Endod 41: 937-942.

23. Duke F, Shen Y, Zhou H, Ruse ND, Wang ZJ, et al. (201) Cyclic Fatigue of ProFile Vortex and Vortex Blue Nickel-Titanium Files in Single and Double Curvatures. J Endod 41: 1686-1690.

24. VDW Disponible en: https://www.vdw-dental.com/sortiment/ produktdetail/reciproc-blue-feilen/Alemania 2017.

25. Yáñez A (2017) Nuevas Aleaciones Reciproc ${ }^{\circledR}$ Blue: "Una nueva lima, aún mejor". Revista de la Sociedad de Endodoncia de Chile N35.

26. VDW GmbH. Reciproc ${ }^{\circledR}$ blue User Guide. $1^{\circ}$ Edición. Setiembre 2016.

27. De-Deus G, Silva EJ, Vieira VT, Belladonna FG, Elias CN, et al. (2017) Blue Thermomechanical Treatment Optimizes Fatigue Resistance and Flexibility of the Reciproc Files. J Endod 43: 462-466. 